\title{
A Multi-year Analysis of Decision Fatigue in Opioid Prescribing
}

\author{
Jordan Hughes, $B A^{7}$ (D) Jerzy Lysikowski, $P h D^{2}$, Rabina Acharya, $\mathrm{MPH}^{2}$, \\ Eleanor Phelps, $R N^{3}$, and Enas Kandil, MD MSc ${ }^{4}$
}

'Medical School, University of Texas Southwestern Medical Center, Dallas, TX, USA; ${ }^{2}$ Office of Medical Education, University of Texas Southwestern Medical Center, Dallas, TX, USA; ${ }^{3}$ Office of Quality, Safety, and Outcomes Education, University of Texas Southwestern Medical Center, Dallas, TX, USA; ${ }^{4}$ Department of Anesthesiology \& Pain Management, University of Texas Southwestern Medical Center, Dallas, TX, USA.

J Gen Intern Med 35(4):1337-9

DOI: $10.1007 / \mathrm{s} 11606-019-05217-\mathrm{x}$

(c) Society of General Internal Medicine 2019

\section{INTRODUCTION}

Decision fatigue is a psychological phenomenon describing how people can, when making a series of decisions, deplete their mental resources over time and unknowingly attempt to reduce their cognitive burden by favoring the status quo - the cognitively "easier" choice. ${ }^{1}$ Every day, primary care physicians (PCPs) make myriad decisions which subject them to such mental depletion. As one study of the phenomenon in primary care concludes, "decision fatigue progressively impairs clinicians' ability to resist ordering inappropriate treatments."

Because the USA faces an unprecedented epidemic of opioid abuse, we must understand the extent to which decision fatigue affects opioid prescribing, as the prescription of these drugs has been associated with both long-term use and overdose deaths. ${ }^{3-5}$ Additionally, it is important to measure how recent opioid interventions have, if at all, affected decision fatigue's role. The aim of this study is to measure PCPs' varying likelihoods of prescribing opioids throughout the clinical day, before and after major interventions were implemented to combat the epidemic, as this can serve as an indication of both the presence of decision fatigue and the impact of concerted interventions.

\section{METHODS}

We began by selecting the years 2014 and 2017 to represent the pre- and post-intervention periods for study, as many major interventions to combat the opioid epidemic took place in 2016. These interventions include new clinical practice guidelines, federal and state legislation, and the severity of the epidemic becoming prominent national news. Next, we analyzed the percentage of appointments in which opioids were

Received June 25, 2019

Accepted July 12, 2019

Published online July 31, 2019 prescribed in each hour of physicians' clinical days, at three exclusively primary care clinics at University of Texas Southwestern Medical Center. Scheduled appointment times were used as substitutes for visit times. We then excluded patients with cancer and those who had surgery within 6 weeks of an appointment, in order to minimize the number of appointments in which opioids may be prescribed by clear clinical indication. This study was exempt from review by the UT Southwestern Institutional Review Board.

We employed logistic regression analysis to determine the predictive relationship between appointment time and opioid prescriptions, using physicians' prescription rates in their first clinic hour as the reference for calculating odds ratios in each year.

\section{RESULTS}

A total of 34,972 clinic visits in 2014 and 42,313 clinic visits in 2017 met our inclusion criteria (Table 1). The overall likelihood of patients being prescribed opioids in 2017 was $22.5 \%$ less than in 2014 (95\% CI, 0.732-0.822). However, the hourly likelihoods of patients being prescribed opioids increased significantly throughout the clinical day in each year (Fig. 1).

\section{DISCUSSION}

While there was a significant decrease in the overall likelihood of being prescribed opioids in 2017 compared with 2014 , the variation in hourly prescription likelihoods is similar in both years. These results reflect the findings of similar studies of prescription decision fatigue., ${ }^{2} 6$ The results show that while interventions to combat the opioid epidemic were successful in reducing the overall amount of opioids prescribed, they had minimal impact on the effect of decision fatigue. Additionally, we had hypothesized that by increasing regulation and social pressure to not prescribe opioids in the midst of this epidemic, the cognitively "easier" choice for physicians in 2017 would change to become not prescribe, and thus prescription 
Table 1 Characteristics of Primary Care Visits in 2014 and 2017. Comparisons Between the Years 2014 and 2017 in Each Characteristic Category (Patient Age, Patient Sex, Clinic Site, Physician Sex) Have $P$ Values $<0.001$

2014

\section{Characteristic}

Patient age group, no. (\%)

$18-64$

$>65$

Patient sex, no. (\%)

Male

Female

Unknown

Clinic site, no. (\%)

General internal

medicine

Family medicine

Geriatric medicine

Physician sex, no. (\%)

Male

Female

Unknown

\section{Characteristic}

Patient age group, no. (\%)

18-64

$>65$

Patient sex, no. (\%)

Male

Female

Unknown

Clinic site, no. (\%)

General internal

medicine

Family medicine

Geriatric medicine

Physician sex, no. (\%)

Male

Female

Unknown
Overall sample $(N=$ 34,972)

22,576

12,396

12,446

22,520

6

22,677

9683

2612

$\mathbf{3 4 , 3 8 2}($ missing $=\mathbf{5 9 0})$

12,458

21,923

1

2017

Overall sample $(N=$

42,313)

42,307 (missing $=6$ )

26,240

16,067

14,259

28,053

1

26,624

11,381

4308

42,237 (missing $=76$ )

8260

33,977
Prescribed opioid (1866 [5.34\%])

$1173(5.20 \%)$

$693(5.59 \%)$

$569(4.57 \%)$

$1297(5.76 \%)$

$0(0.00 \%)$

$1308(5.77 \%)$

$441(4.55 \%)$

$117(4.48 \%)$

1841

$588(4.72 \%)$

$1253(5.72 \%)$

$0(0.00 \%)$

Prescribed opioid (1838 [4.34\%])

$1192(4.54 \%)$

$645(4.01 \%)$

$561(3.93 \%)$

$1277(4.55 \%)$

$0(0.00 \%)$

$1343(5.04 \%)$

$358(3.15 \%)$

$137(3.18 \%)$

1838

$310(3.75 \%)$

$1528(4.50 \%)$
Not prescribed opioid $(33,106$

[94.66\%])

$21,403(94.80 \%)$

$11,703(94.41 \%)$

$11,877(95.43 \%)$

$21,223(94.24 \%)$

$6(100 \%)$

$21,369(94.23 \%)$

$9242(95.45 \%)$

$2495(95.52 \%)$

$\mathbf{3 2 , 5 4 1}$

$11,870(95.28 \%)$

$20,670(94.28 \%)$

$1(100 \%)$

Not prescribed opioid $(40,475$

[95.66\%])

$25,048(95.46 \%)$

$15,422(95.99 \%)$

$13,698(96.07 \%)$

$26,776(95.45 \%)$

$1(100 \%)$

$25,281(94.96 \%)$

$11,023(96.85 \%)$

$4171(96.82 \%)$

$\mathbf{4 0 , 3 9 9}$

$7950(96.25 \%)$

$32,449(95.50 \%)$

$P$ value

0.12

$<0.001$

$<0.001$

$<0.001$

$-$
$<0.001$

$P$ value

0.01

0.003

0.002

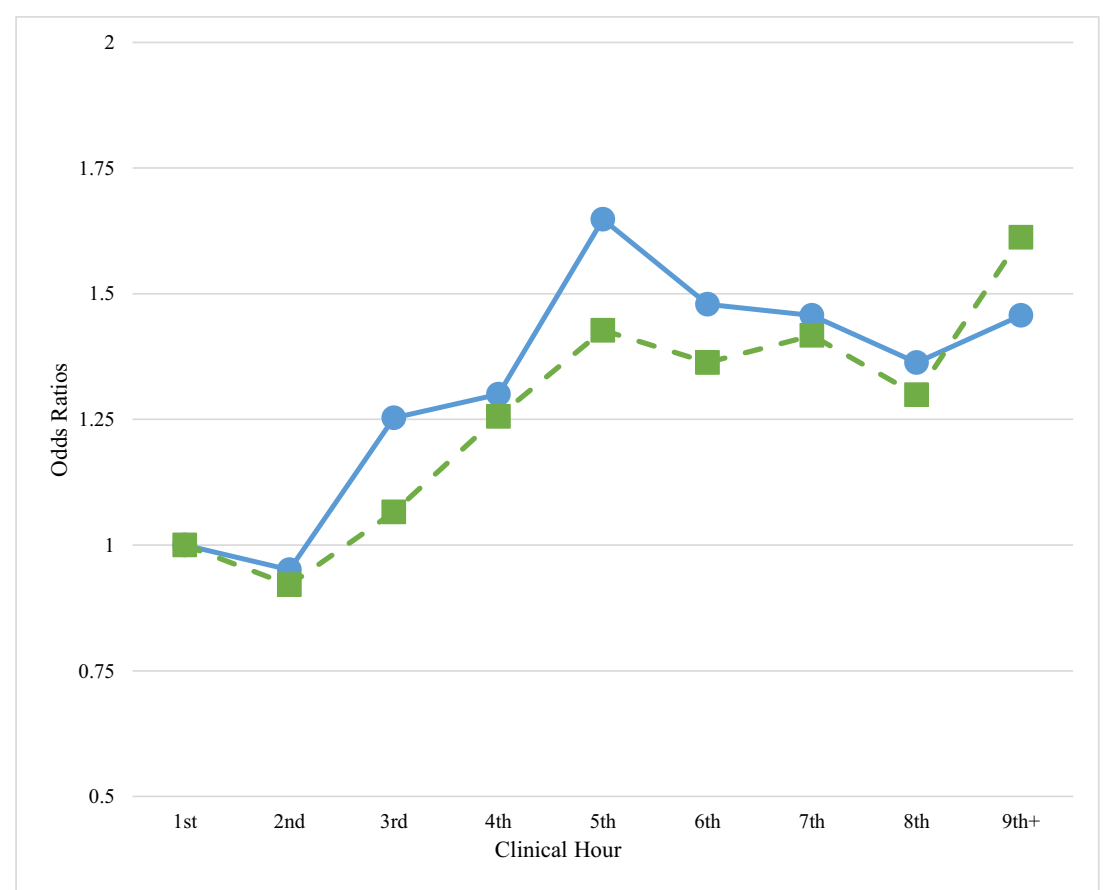

Figure 1 Hourly opioid prescribing likelihoods in 2014 and 2017. The hourly odds ratios for the year 2014 are in blue; those for the year 2017 are in green. The hourly odds ratios, with $95 \%$ confidence intervals in parentheses, for 2014 are as follows: 1 (reference), 0.951 (0.752-1.203), 1.253 (0.999-1.573), 1.300 (1.036-1.632), 1.648 (1.296-2.096), 1.479 (1.152-1.899), 1.457 (1.150-1.846), 1.363 (1.069-1.737), 1.457 (1.141-1.859). The hourly odds ratios, with $95 \%$ confidence intervals in parentheses, for 2017 are as follows: 1 (reference), $0.921(0.736-1.153)$, 1.066 (0.8571.326), 1.256 (1.012-1.559), 1.427 (1.132-1.798), 1.363 (1.065-1.743), 1.417 (1.128-1.780), 1.299 (1.026-1.645), 1.612 (1.281-2.029). 
likelihood would decrease throughout the clinical day. This hypothesis is not supported by the results of the study.

This study has multiple limitations, including that it was performed at a single academic medical center and other factors besides decision fatigue may be contributing to our results. Future studies may include measuring the effect of implementing decision support tools for opioid prescribing into clinical practice, as well as continuing to monitor prescription likelihoods as patient and physician perceptions of opioids continue to change.

Acknowledgments: The authors would like to thank the Office of Quality, Safety, and Outcomes Education at the University of Texas Southwestern Medical Center for their contributions of funding, data analysis, and manuscript review.

Corresponding Author: Jordan Hughes, BA; Medical School, University of Texas Southwestern Medical Center, Dallas, TX, USA (e-mail: jordan.bland@utsouthwestern.edu).

\section{Compliance with Ethical Standards:}

Conflict of Interest: The authors declare that they do not have a conflict of interest.

\section{REFERENCES}

1. Danziger S, Levav J, Avnaim-Pesso L. Extraneous factors in judicial decisions. Proc Natl Acad Sci U S A 2011;108(17):6889-6892. doi:https:// doi.org/10.1073/pnas.1018033108

2. Linder JA, Doctor JN, Friedberg MW, et al. Time of Day and the Decision to Prescribe Antibiotics. JAMA Intern Med 2014;174(12):2029-2031. doi:https://doi.org/10.1001/jamainternmed.2014.5225

3. What is the U.S. Opioid Epidemic? U.S. Department of Health and Human Services. https://www.hhs.gov/opioids/about-the-epidemic/. Last reviewed January 22, 2019. Accessed 4 March 2019.

4. Shah A, Hayes CJ, Martin BC. Characteristics of Initial Prescription Episodes and Likelihood of Long-Term Opioid Use — United States, 20062015. MMWR Morb Mortal Wkly Rep 2017;66:265-269. doi:https://doi. org/10.15585/mmwr.mm6610al

5. Bohnert ASB, Valenstein M, Bair MJ, et al. Association Between Opioid Prescribing Patterns and Opioid Overdose-Related Deaths. JAMA 2011;305(13):1315-1321. doi:https://doi.org/10.1001/jama.2011.370

6. Philpot, LM, Khokhar BA, Roellinger DL, et al. Time of Day is Associated with Opioid Prescribing for Low Back Pain in Primary Care. J Gen Intern Med 2018;33(11):1828-1830. doi:https://doi.org/10.1007/s11606-0184521-8

Publisher's Note Springer Nature remains neutral with regard to jurisdictional claims in published maps and institutional affiliations. 